\title{
Risikoschwangerschaften Möglichkeiten der Pränataldiagnostik
}

Elke Bäz • Beatrix Brinckwirth

Nicht jede Schwangerschaft verläuft komplikationslos. Manche Risiken für Mutter und Kind lassen sich durch die Pränataldiagnostik jedoch schon früh erkennen. So bietet sich die Chance, rechtzeitig medizinische Hilfe zu leisten - und mütterliche wie kindliche Morbidität zu vermindern. In der Schwangerschaftsvorsorge ist zu unterscheiden zwischen der Basisuntersuchung per Ultraschall in der frauenärztlichen Betreuung - und der differenzierten Diagnostik, ggf. inklusive invasiver Verfahren beim Pränatalmediziner. Für alle Untersuchungen gilt aber: Die psychischen Belastungen und Konsequenzen eines auffälligen Befundes sind für die Eltern gravierend. Pränataldiagnostiker müssen daher speziell geschult sein und interdisziplinär beraten.

Definition Unter pränataler Diagnostik (PND) versteht man vorgeburtliche Untersuchungen des Ungeborenen, die Aussagen über bestimmte Krankheiten des ungeborenen Kindes sowie mögliche Gefahren für Leben und Gesundheit der Schwangeren und des Ungeborenen zulassen. So kann man ggf. eine Therapie einleiten, um den Ausgang der Schwangerschaft zu verbessern.

Häufigkeit von Fehlbildungen Etwa 4\% aller neugeborenen Kinder haben angeborene Erkrankungen, die nur teilweise außerhalb spezialisierter Pränatalambulanzen im Rahmen der frauenärztlichen Ultraschalluntersuchungen erkannt werden können. Zu den häufigsten angeborenen Fehlbildungen zählen:

- Herzfehler (bis zu 1\%)

- Fehlbildungen der Nieren und Harnwege (1\%)

- Chromosomenfehler (0,5\%)

Nutzen der Pränataldiagnostik Es gilt heute als unstrittig, dass das vorgeburtliche Wissen um eine Erkrankung den Lebensstart eines Neugeborenen deutlich verbessern kann:

- Manche Fehlbildungen (z.B. spezielle Herzfehler, Zwerchfellhernie, Neuralrohr- oder Bauchwanddefekte) erfordern eine Operation innerhalb der ersten Lebenstage.

- Hier kann die Vorbereitung auf die Geburt eines kranken Kindes für die werdenden Eltern hilfreich und für das Kind lebensrettend sein.
Wichtigstes Instrument: Ultraschall Schwerpunkt der PND liegt auf der detaillierten Ultraschalluntersuchung.

- Zum einen kann man die mütterlichen Schwangerschafts- und Geburtsrisiken reduzieren (z.B. Verzicht auf Sectio bei infauster fetaler Prognose oder primäre Sectio bei Plazenta prävia).

- Zum anderen lassen sich Schäden des Neugeborenen mindern, wenn man Risiken (z.B. plazentare Mangelversorgung oder angeborene Fehlbildung/Erkrankung) erkennt und ggf. entsprechend behandelt (z.B. intrauterine fetale Bluttransfusion oder vorzeitige Entbindung).

Ergebnis auffällig - und dann? In den letzten 20 Jahren haben sich die Handlungsoptionen im Rahmen der PND erweitert:

- von der Entscheidung zwischen Abbruch vs. Fortsetzen der Schwangerschaft

- um die Möglichkeiten aktiver (medikamentöser und chirurgischer) Interventionen zur Verbesserung des Langzeit-Outcomes des Fetus.

Pränataldiagnostik ist interdisziplinär Pränataldiagnostiker kooperieren eng mit den betreuenden niedergelassenen Frauenärzten und Hebammen, mit den Kollegen der Geburtsmedizin, den Neonatologen und anderen Fachrichtungen der Kinderheilkunde - u.a. der Kinderkardiologie, -nephrologie, -neurologie und -chirurgie. Weitere Schnittstellen gibt es mit der Humangenetik und Reproduktionstoxikologie ( $\bullet$ Kasten S. 238) sowie mit psychologischen Beratungsund Begleitungsangeboten.

\section{Die gesetzliche Mutterschaftsvorsorge $\nabla$}

Ziel der Vorsorge Bereits die gesetzlich geregelte ärztliche Schwangerschaftsbetreuung ist als pränatale Diagnostik anzusehen, die gewisse Basisanforderungen erfüllen soll:

„Durch die ärztliche Betreuung während der Schwangerschaft und nach der Entbindung sollen mögliche Gefahren für Leben und Gesundheit von 
Mutter oder Kind abgewendet sowie Gesundheitsstörungen rechtzeitig erkannt und der Behandlung zugeführt werden. Vorrangiges Ziel der ärztlichen Schwangerenvorsorge ist die frühzeitige Erkennung von Risikoschwangerschaften und Risikogeburten“ [1].

Routineuntersuchungen Im Rahmen der Mutterschaftsvorsorge sind in Deutschland für unkomplizierte Schwangerschaften 3 Ultraschalluntersuchungen vorgesehen:

- 1. Screening: von Beginn der 9. bis zum Ende der 12. Schwangerschaftswoche (SSW)

2. Screening : von Beginn der 19. bis zum Ende der 22. SSW

- 3. Screening: von Beginn der 29. bis zum Ende der 32. SSW

Das 1. Screening Bislang werden bei der ersten Routineuntersuchung (9.-12. SSW) der Sitz der Schwangerschaft (intra- oder extrauterin), die Vitalität und Anzahl der Feten, bei Mehrlingen die Chorion- und Amnionverhältnisse, das Alter der Schwangerschaft anhand der Schädel-Steiß-Länge sowie die körperliche Integrität (Ausschluss grobstruktureller Fehlbildungen) untersucht.

Das 2. Screening Für die Untersuchung der fetalen Morphologie ist vor allem der Ultraschall im 2. Trimenon relevant $(18+0$ bis $21+6$ SSW $)$. Er dient dazu, die Biometrie des Fötus zu erfassen und eventuelle Hinweiszeichen für Entwicklungsstörungen zu erkennen ( Tab. 1). Da die bisherigen Vorgaben einen weiten Interpretationsspielraum zulassen, soll gemäß einer geplanten Erweiterung der gesetzlichen Vorgaben schon bei der Routineuntersuchung nach Fehlbildungen/ fetalen Zuständen gesucht werden, die

- mit dem Leben nicht vereinbar sind

- mit hoher Morbidität bzw. Langzeitbehinderung einhergehen

- das Potenzial für eine intrauterine Therapie beinhalten

- eine postnatale Untersuchung oder Behandlung erfordern.

Es ist geplant, Schwangeren die Option zu eröffnen, im 2. Trimenon zwischen einer biometrischen Sonografie mit oder ohne systematische Untersuchung der fetalen Morphologie durch einen besonders qualifizierten Untersucher zu wählen.

Diese erweiterte Ultraschalluntersuchung soll dann ebenfalls zum Programm der Mutterschaftsvorsorge gehören, aber nicht Bestandteil des Screenings sein. Sie soll nicht auf die Abklärung genetischer Eigenschaften ausgerichtet sein und damit nicht in den Anwendungsbereich des Gendiagnostikgesetzes (GenDG, o Kasten S. 239) fallen [2].
Das 3. Screening Das Basis-Screening im 3. Trimenon von Beginn der 29. bis zum Ende der 32. SSW umfasst die Kontrolle auf mögliche Hinweiszeichen für Entwicklungsstörungen gemäß dem 2. Screening.

\section{Differenzierte Pränataldiagnostik}

Für wen? Im Rahmen der Mutterschaftsvorsorge sollen Risikoschwangerschaften und mögliche Risikogeburten erkannt und besonders eng überwacht werden. Dazu gehören

- Schwangerschaften, bei denen aufgrund der Vorgeschichte oder erhobener Befunde mit einem erhöhten Risiko für Leben und Gesundheit von Mutter oder Kind zu rechnen ist.

Typische Risikokonstellationen Häufige Indikationen für die Überweisung zum Spezialisten der Pränataldiagnostik, ggf. bei fortgeschrittener Schwangerschaft (>24. SSW) in Kooperation mit einem Perinatalzentrum, sind:

- mütterliche Risikofaktoren/Komplikationen

$\triangleright$ maternale Vorerkrankungen (wie Hypertonus, Autoimmunerkrankung, Z.n. Transplantation, Z.n. korrigiertem Herzfehler, Nieren-/ Lebererkrankung, Adipositas, Diabetes mellitus u.v.m.)

$\triangleright$ Medikamenteneinnahme, Strahlenexposition

$\triangleright$ Kosanguinität, Genträger, belastete Familienanamnese

$\triangleright$ vorangegangene Schwangerschaftskomplikationen: Frühgeburt, totgeborenes oder geschädigtes Kind, Entbindungen von Kindern über $4 \mathrm{~kg}$ oder hypotrophen Kindern (small for date babies)

$\triangleright$ Z.n. wiederholten Aborten

$\triangleright$ maternale Infektionen (materno-fetale Transmission, z.B. CMV-, Toxoplasmose-, Parvovirus-B19-Infektion)

$\triangleright$ maternale irreguläre Antikörper, z.B. Blutgruppen-Inkompatibilität (Morbus haemolyticus fetalis bzw. neonatorum), antinukleäre Antikörper (Risiko der Störung des fetalen kardialen Reizleitungssystems mit fetalen Brady- oder Tachy-Arrythmien)

$\triangleright$ Zeichen der Entwicklung einer schweren Präeklampsie (ehemals EPH-Gestose), dar-

\section{Fokus des Basisscreenings im 2. Trimenon}

- Fruchtwassermenge

- körperliche Entwicklung

- Körperumriss

- fetale Strukturen

- Herzaktion

- Bewegungen

Plazentalokalisation und -struktur
Tab. 1 Morphologische, strukturelle und physiologische Parameter, die im Fokus des Ultraschalls im 2. Trimenon stehen. Bestimmte Veränderungen stellen sonografische Hinweiszeichen für Entwicklungsstörungen dar. 
Abb. 1 Fetale Echokardiografie. Oben: Fetus in der 14. SSW. Die Aufnahme zeigt eine (noch meist physiologische) Tricuspidalinsuffizienz.

Unten: Beispiel eines Dobble outlet aus dem rechten Ventrikel (Fetus in der 28. SSW).
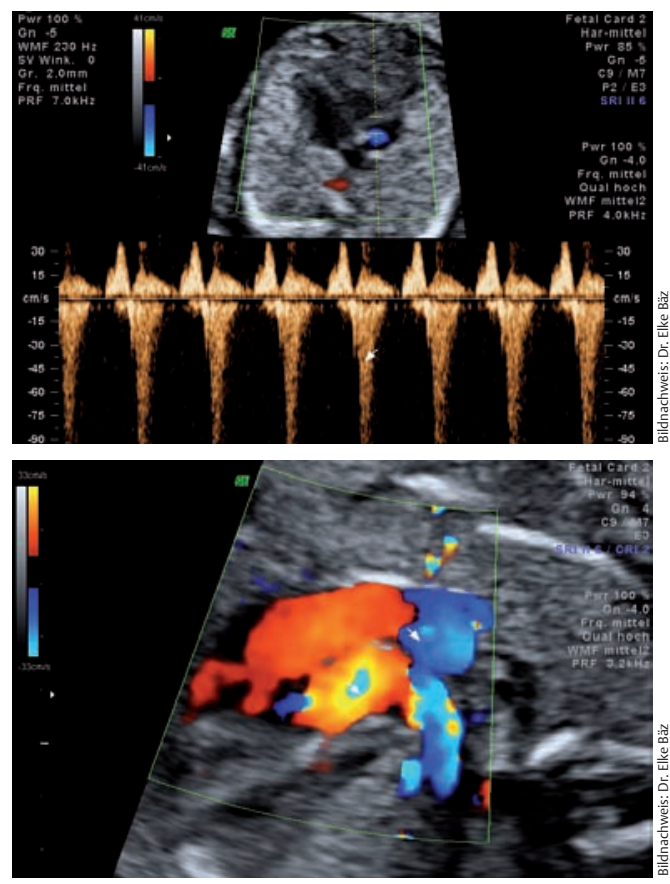

unter Blutdruck $\geq 140 / 90 \mathrm{mmHg}$, Eiweißausscheidung $\geq 1 \mathrm{~g} / 24 \mathrm{~h}$ (s. Beitrag OS. 244)

- Kindliche Auffälligkeiten oder Risiken $\triangleright$ sonografischer Verdacht auf Fehlbildung

$\triangleright$ Größendiskrepanz zum Gestationsalter

$\triangleright$ veränderte Fruchtwassermenge

$\triangleright$ Mehrlinge

Früherkennung von Chromosomenfehlern bei „älteren“ Schwangeren Die erste Amniozentese zum Nachweis von Chromosomenaberrationen wurde in Deutschland 1969 durchgeführt. Nach den aktuellen Mutterschafts-Richtlinien gilt jede Schwangere ab 35 Jahren als Risikoschwangere - und soll an einen Spezialisten überwiesen werden, der eine Fruchtwasseruntersuchung oder Chorionzottenbiopsie durchführen kann. Der Grund:

- Mit dem Alter der Mutter steigt das Risiko für Chromosomenfehler (Aneuploidie).

Als die Mutterschafts-Richtlinien 1985 in Kraft traten, waren nur etwa 5\% der Schwangeren in Deutschland älter als 35 Jahre - konzipierten aber $30 \%$ der Feten mit Chromosomenfehler.
Heute ist jede 4. Schwangere älter als 35 Jahre. Behielte man dieses Alter als cut-off für die Amniozentese bei, wäre die Screening-Population unverhältnismäßig hoch.

Seit etwa 1998 hat sich das sogenannte Ersttrimester-Screening (s. unten) etabliert. Damit steht jeder Schwangeren (nicht nur den > 35-Jährigen) eine individuelle Entscheidungshilfe für oder gegen eine invasive Diagnostik zum Ausschluss fetaler Chromosomenfehler zur Verfügung [3, 6].

\section{Keine Diagnostik ohne Aufklärung und Beratung!}

Ärztliche Beratung vor PND Im Sinne des GenDG stellen die differenzierte PND und auch die vorgeburtliche Risikoabklärung eine genetische Diagnostik dar ( 0 Kasten S. 239).

- Vor PND hat der verantwortliche Arzt die Schwangere über Wesen, Bedeutung und Tragweite der (genetischen) Untersuchung aufzuklären. Die Aufklärung umfasst insbesondere

$\triangleright$ Zweck, Art, Umfang und Aussagekraft der Untersuchung einschließlich der erzielbaren Ergebnisse

$\triangleright$ gesundheitliche Risiken, die mit der Kenntnis des Untersuchungsergebnisses und mit der erforderlichen Probenahme für den Fötus verbunden sind.

- Nach der Aufklärung ist der Schwangeren eine angemessene Bedenkzeit bis zur Entscheidung über die Einwilligung einzuräumen.

Psychosoziale Beratung und Begleitung Die PND mit ihren diagnostischen und therapeutischen Verfahren stellt Schwangere und deren Partner manchmal vor schwierige Entscheidungen. Hier ist es hilfreich, neben der medizinischen Beratung auch soziale und psychische Aspekte zu besprechen.

- Ziel der psychosozialen Beratung ist es, eine kompetente Entscheidung hinsichtlich der Inanspruchnahme von vorgeburtlichen Untersuchungen zu ermöglichen sowie

- Unterstützung und Begleitung bei den dabei entstehenden Herausforderungen anzubieten.

\section{Exkurs: Reproduktionstoxikologie}

Die Reproduktionstoxikologie befasst sich mit den Auswirkungen von potenziell schädigenden Faktoren (wie Medikamenten, Arbeitsplatzund Umweltchemikalien) auf die Fruchtbarkeit der Eltern und die Entwicklung des Kindes während Schwangerschaft und Stillzeit.

Viele Frauen können während einer Schwangerschaft nicht auf Medikamente verzichten. Das Problem: Die Arzneimittelhersteller geben ihre Produkte aus Angst vor Schadensersatzforderungen nicht für Schwangere frei. Hilfreich für den behandelnden Arzt sind dann spezielle Datenbanksysteme, wie z. B.

O www.reprotox.de oder O www.embryotox.de

Hier werden reproduktionstoxikologische Daten aus der Wissenschaft erfasst. Dadurch stehen aktuelle Erkenntnisse und Informationen über die Folgen einer Medikamenten- oder Schadstoffexposition in Schwangerschaft und Stillzeit sowie über fruchtschädigende Umweltfaktoren zur Verfügung. 
Der Arzt berät die Patientin und stellt auf Wunsch Kontakt zu Schwangeren- und Schwangerschaftskonfliktberatungsstellen her. Dabei sollte er auf die verschiedenen Fragen, die die werdenden Eltern beschäftigen, näher eingehen:

- Welche Untersuchungen sind in der individuellen Lebenssituation sinnvoll?

- Welche Auswirkungen hätte ein auffälliger Befund?

- Welche Optionen zur weiteren Lebensgestaltung stehen offen?

- Welche Möglichkeiten der Frühförderung gibt es?

Ausführliche Anamnese Vor einer differenzierten Pränataldiagnostik erhebt man eine medizinisch-genetische Anamnese. Dabei muss neben der Dokumentation einer eingehenden Eigenund Schwangerschaftsanamnese auch nach Fehlbzw. Totgeburten sowie nach eventuell genetisch bedingten Erkrankungen und Behinderungen in der eigenen und der Familie des Kindsvaters gefragt werden.

„Ergeben sich im Rahmen der Mutterschaftsvorsorge Anhaltspunkte für ein genetisch bedingtes Risiko, so ist der Arzt gehalten, die Schwangere über die Möglichkeiten einer humangenetischen Beratung und / oder humangenetischen Untersuchung aufzuklären“ [1].

Humangenetische Beratung Die genetische Beratung erfolgt allgemein verständlich und ergebnisoffen.

- Zu erörtern sind insbesondere die möglichen medizinischen, psychischen und sozialen Fragen im Zusammenhang mit der Vornahme oder Nichtvornahme einer Untersuchung sowie

- die Angebote zur Unterstützung bei physischen und psychischen Belastungen durch die Untersuchung und das Ergebnis.

Kommen genetische Erkrankungen in der Familie der Eltern vor, sollte der Humangenetiker das potenzielle Wiederholungsrisiko aufzeigen: Bei einer unklaren, wahrscheinlich genetisch bedingten Behinderung eines Kindes des Paares liegt das Risiko geschätzt bei 1:16 und bei einer bekannten autosomal-rezessiven Erkrankung bei 1:4.

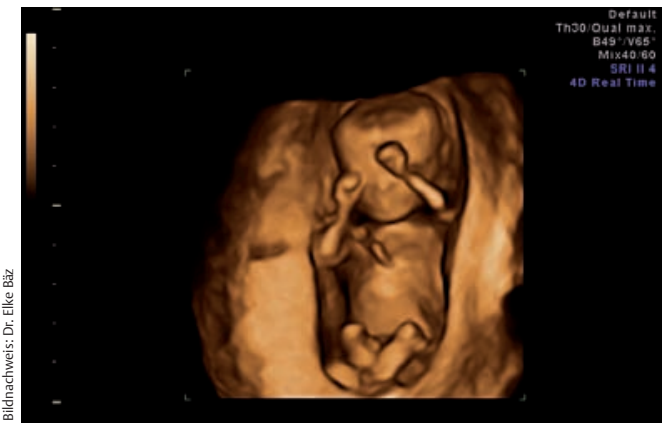

Abb. 2 Fetus $13+3$ SSW, unauffällige Oberflächendarstellung im 3-D-Modus

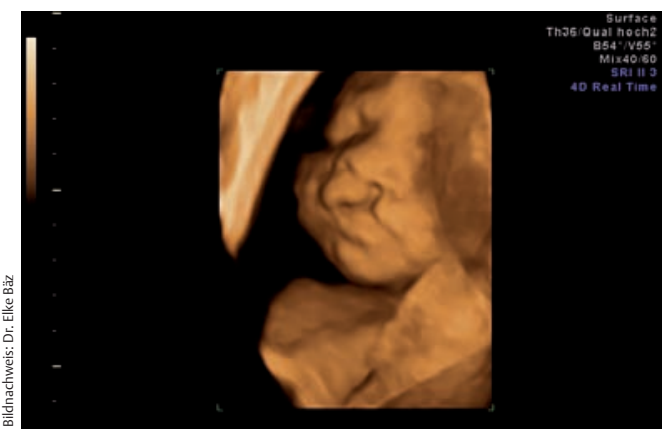

Abb. 3 Fetus mit einseitiger Lippen-Kiefer-Spalte in der 25 SSW, Oberflächendarstellung im sog. 4-D-Modus. Dabei werden auch die Bewegungen des Kindes aufgezeichnet.

\section{Methoden und Zeitpunkt der differenzierten Pränataldiagnostik $\nabla$}

Optionen Nach eingehender Beratung über die Zielsetzung der pränatalmedizinischen Untersuchung erfolgt eine differenzierte Ultraschalluntersuchung mit

- hochauflösender 2-dimensionaler Darstellung fetaler und mütterlicher Strukturen,

- Blutflussdarstellungen, wie fetaler Echokardiografie ( Abb. 1) und Dopplersonografie der maternalen und fetalen Gefäße, sowie

- ggf. 3- (๑ Abb. 2) oder 4-dimensionaler Sonografie (Realtime-3-D, o Abb. 3).

Die Möglichkeiten weiterführender invasiver Eingriffe wie Chorionzottenbiopsie (Plazentagewebsentnahme), Amniozentese (Fruchtwasserentnahme) und bei speziellen Fragestellungen Fetalblutentnahme ergänzen das Spektrum der Pränatalmedizin.

Ultraschall in der Frühschwangerschaft Über lange Zeit lag der Schwerpunkt der PND auf dem Nachweis oder Ausschluss von kindlichen Fehlbildungen im 2. und 3. Trimenon. Nun hat sich ein

\section{Exkurs: Gesetz über genetische Untersuchungen bei Menschen (Gendiagnostikgesetz - GenDG)}

$\S 1$ Zweck dieses Gesetzes ist es, die Voraussetzungen für genetische Untersuchungen [...] zu bestimmen [...], um insbesondere die staatliche Verpflichtung zur Achtung und zum Schutz der Würde des Menschen und des Rechts auf informationelle Selbstbestimmung zu wahren.

$\S 2$ Dieses Gesetz gilt für genetische Untersuchungen und im Rahmen genetischer Untersuchungen durchgeführte genetische Analysen bei geborenen Menschen sowie bei Embryonen und Föten während der Schwangerschaft [...]

(Auszug aus dem Gendiagnostikgesetz vom 31. Juli 2009; BGBI. IS. 2529, 3672)

Da die pränatale Untersuchung des Fötus in den Bereich prädiktiver Untersuchung auf genetische Eigenschaften fällt, regelt das GenDG

- den obligaten Beratungsumfang vor und nach der Diagnostik sowie

- den Umgang mit Proben und Untersuchungsergebnissen zum Schutz der Betroffenen. 

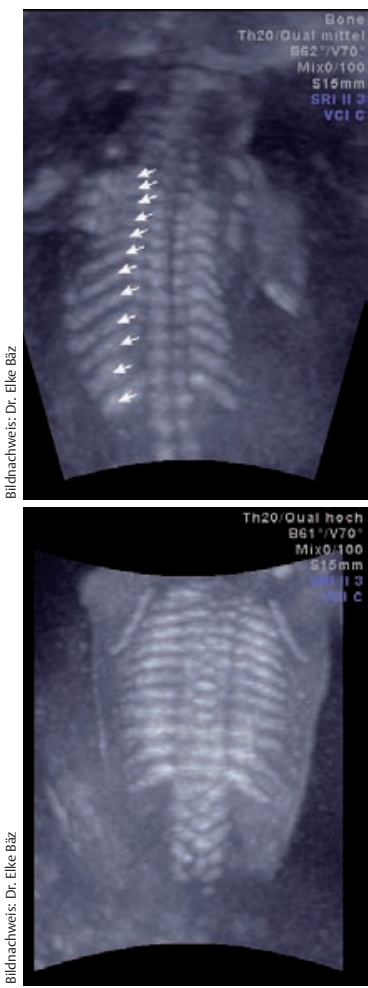

Abb. 4 Thorax-Ultraschallaufnahmen zweier Föten. Oben: Thoraxhypoplasie bei thanatophorer Skelettdysplasie (30. SSW). Unten: fetale normale Skelettdarstellung (20. SSW).
Schwerpunkt der PND auf die Frühschwangerschaft verlagert.

- Im Rahmen der Ultraschalldiagnostik im 1. Trimenon (sog. Erst-Trimester-Screening) sollen anhand eines Algorithmus die Weichen gestellt werden für eine intensivierte vs. eine RoutineSchwangerschaftsbegleitung [4].

- Diese gezielte Fehlbildungsdiagnostik nehmen Untersucher mit entsprechender Erfahrung vor.

Eingeschränkte Aussagekraft Bei der Untersuchung in der Frühschwangerschaft sollte man immer berücksichtigen, dass Erkrankungen, die sich intrauterin erst ausbilden, nicht in jedem Fall in dieser frühen Schwangerschaftsphase diagnostizierbar und damit auszuschließen sind. Ferner ist der spätere Erkrankungsverlauf oft nicht abschätzbar und damit eine Prognose nicht möglich - so z. B. bei obstruktiven Uropathien und konsekutiver Nierenschädigung, Anhydramnie mit sich entwickelnder Lungenhypoplasie oder bei hämodynamisch wirksamen Herzfehlern mit konsekutiver Myokardhypertrophie.

Nur eine gesicherte Diagnose einer fetalen Erkrankung kann Grundlage von Überlegungen gegen eine Fortsetzung einer Schwangerschaft sein.

Ersttrimester-Screening Die verbesserte Ultraschalltechnik ermöglicht es, die fetale Anatomie schon im 1. Trimenon detailliert zu untersuchen. Grob-strukturelle Auffälligkeiten wie Anenzephalus, Holoprosenzephalie, Enzephalozelen, Extremitätenfehlbildungen und andere morphologische Auffälligkeiten des Fötus sind ab der 11. SSW erkennbar.

- Dabei hat die subkutane Transparenz zwischen Haut und Weichgewebe über der zervikalen Wirbelsäule des Fetus, die sog. Nackentransparenz, große Bedeutung: Eine zunehmende Weite dieser Flüssigkeitsansammlung korreliert mit möglichen fetalen Erkrankungen ( $\bullet$ Tab. 2). Das Ersttrimester-Screening erfolgt standardisiert und ist an Qualitätskontrollen gebunden.

- In Kombination mit dem Alter und den Serumparametern der Mutter bietet es eine fundierte (NT) können vielfältig sein (Angaben aus [5]). vorgeburtliche Risikoabklärung für mögliche numerische Chromosomenanomalien (Trisomie 21,13,18) beim Fötus: So kann das individuelle Risiko mit einer Sensitivität von bis zu 95\% bei einer Falschpositivrate von unter 5\% spezifiziert werden.

Damit kann man Schwangeren jeden Alters eine Entscheidungshilfe für oder gegen eine invasive Diagnostik anbieten.

\section{Invasive Diagnostik: Chorionzottenbiopsie}

Gibt es vor der 14+0 SSW Verdacht oder erhöhte Risiken für das Vorliegen einer Chromosomenstörung oder genetischen Erkrankung, lässt sich dies ab 11+0 SSW durch eine Chorionzottenbiopsie unter Ultraschallsicht abklären.

- Dabei aspiriert man mit einer Nadel (transabdominal)

- oder mittels Katheter (transvaginal) winzige Gewebsanteile aus der Plazenta.

Die darin enthaltenen Zellen teilen sich spontan und können für eine rasche Analyse verwendet werden.

Invasive Diagnostik: Amniozentese Eine Fruchtwasserentnahme sollte nicht vor der $14+0$ SSW, nach anglo-amerikanischen Leitlinien erst nach 15+0 SSW stattfinden.

- Die Entnahme erfolgt mittels einer Nadel transabdominal unter Ultraschallsicht.

Das Fruchtwasser enthält abgeschilferte fetale Zellen (Haut und Urogenitaltrakt). Diese wachsen in Kultur langsamer als das Plazentagewebe, sodass für eine Analyse erst nach etwa 2 Wochen genügend Zellen zur Verfügung stehen.

Beide invasive Verfahren - Chorionzottenbiopsie wie Amniozentese - bergen ein etwa 0,5\%iges Risiko für das Auslösen einer Fehlgeburt. Dabei ist jedoch zu bedenken, dass auch das natürliche Fehlgeburtsrisiko noch zwischen der 12. und 16. SSW auf ca. 0,5-0,3\% abfällt. Ein maternaler Bluttest wäre hier zwar risikolos, stellt derzeit aber (noch) keine Alternative dar (O Kasten S. 242).

„Organ-Feindiagnostik“ im 2. Trimenon Einige Anomalien sind im frühen Stadium der Schwangerschaft noch nicht erkennbar - darunter ZNSFehlbildungen oder manche Herzfehler. Um derartige Fehlbildungen frühzeitig zu detektieren, folgt dem Ersttrimester-Ultraschall eine detaillierte Sonografie in der 20.-22. SSW. Sie ergänzt den „Routine-Organultraschall“ beim Frauenarzt.

Detaillierte Sonografie 3. Trimenon Der differenzierte Ultraschall nach der 28. SSW hat den Vorteil, dass man neben sonomorphologischen Entwicklungsstörungen, v.a. von Herz und Nieren, auch die Weiterentwicklung zerebraler Strukturen beurteilen kann ( $\bullet$ Abb. 5). 
- So können beispielsweise Stoffwechseldefekte zu einem Hydrops fetalis führen, der sich erst im 3. Trimenon entwickelt.

- Ebenso gibt es Wachstumsretardierungen und kritische fetale Unterversorgungssituationen, die erst im 3. Trimenon erkennbar werden.

\section{Vorgehen bei fetalen Auffälligkeiten \\ $\nabla$}

Eingehende Information und Beratung Stellt man im Rahmen der PND Auffälligkeiten fest, so ist oberstes Ziel, die Schwangere und ihren Partner bestmöglich über die zu erwartende Erkrankung oder Beeinträchtigung des Kindes zu informieren. Dies umfasst Informationen bzw. Kontaktvermittlung zu

- psychosozialer Begleitung

- Gendiagnostikgesetz (Entscheidungsmöglichkeit für/gegen eine genetische Untersuchung sowie Bestimmung des Umgangs mit den Ergebnissen und deren Weitergabe)

- humangenetischer Beratung

- invasiver Diagnostik, pränataler Therapie, vorzeitiger Entbindung

- interdisziplinärer Beratung (Kinderärzte, Selbsthilfegruppen)

Bei Anämie: intrauterine Transfusion Die älteste pränatale Therapie ist die intrauterine fetale Transfusion bei fetaler Anämie. Maternale Laborparameter (ansteigende Antikörper-Titer) und fetale Doppler-Parameter (Blutflussgeschwindigkeiten) können sonografisch die Indikation für eine fetale Blutuntersuchung durch Punktion der Nabelvene geben. Im Rahmen einer Nabelvenenpunktion erfolgt dann auch eine fetale Bluttransfusion.

Problematische Blutversorgung bei Zwillingen Hauptindikation für eine intrauterine Laser-Koagulation ist das Zwillingstransfusionssyndrom (TTS), das bei etwa $15 \%$ der monochorialen Gemini auftritt. Über kommunizierende Gefäße in ihrer gemeinsamen Plazenta kommt es zu einem unbalancierten Blutaustausch zwischen beiden Feten mit resultierender Anämie beim Donor und Polyzythämie beim Rezipienten. Polyhydramnie und Herzinsuffizienz bei Rezipienten und Anhydramnie, Wachstumsretardierung und Hypoxämie beim Donor bedeuten für beide ein deutlich erhöhtes Mortalitäts- und Morbiditätsrisiko. Eine intrauterine Laser-Koagulation versucht die Ursache zu beseitigen, indem die verbindenden Blutgefäße auf der Plazenta unterbrochen werden und damit der Blutfluss vom Donor zum Rezipienten gestoppt wird.

Fetale endoskopische Tracheal-Okklusion (Fetendo-PLUG) bei Zwerchfellhernien Eine kongenitale Zwerchfellhernie ist aufgrund der resultierenden Lungenhypoplasie und pulmonalen

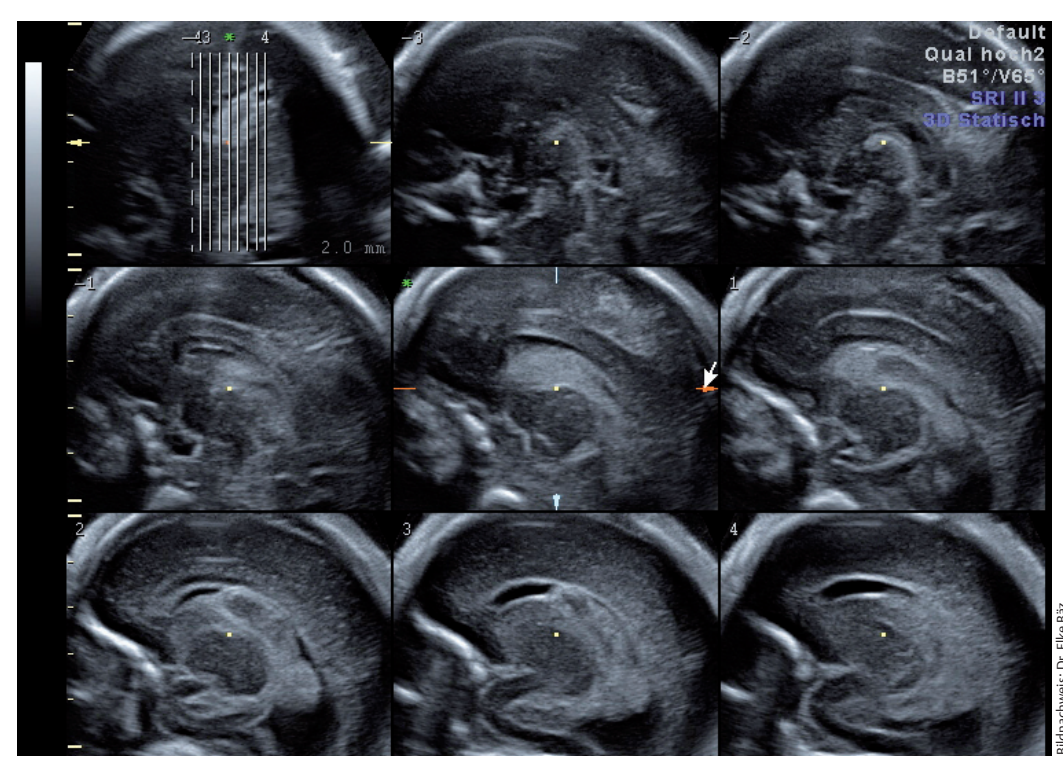

Abb. 5 Sonografische Untersuchung des fetalen Gehirns

Hypertension mit einer hohen postnatalen Morbidität und Mortalität assoziiert.

- Eine intrauterine fetale tracheale Okklusion ist die therapeutische Option, um intrauterin das fetale Lungenwachstum zu stimulieren [7].

Intrauterine chirurgische Therapie bei Spina bifida Der Vergleich eines pränatalen operativen Verschlusses einer Myelomeningozele mit einer operativen Korrektur postnatal kann in einer besseren neurologischen Funktion resultieren. Nachteile jedes pränatalen operativen Vorgehens sind jedoch ein erhöhtes Risiko für Frühgeburt und uterine Narbendehiszenz [8].

\section{Vorgehen bei infauster Prognose \\ $\nabla$}

Schwangerschaft fortführen oder abbrechen?

Die Entscheidung für oder gegen ein Austragen des Kindes benötigt Informationen und Zeit. Wollen die Eltern bei infauster Prognose (z.B. Trisomie 13 und 18, nicht mit dem Leben zu vereinbarende Fehlbildungen) die Schwangerschaft fortführen, kann eine Sterbebegleitung postpartal vorbereitet werden.

Bei infauster Prognose hat die spontane Entbindung, vorzeitig oder am Termin, oberste Priorität.

Seelischer Beistand Begleitungsangebote für Eltern, die ihr Kind - unabhängig von der Schwangerschaftswoche - verloren haben, können den Eltern helfen, den Verlust zu verarbeiten und wieder in einen geregelten Lebensrhythmus hineinzufinden.

- Kontaktadressen vermitteln die Schwangerschaftsberatungsstellen (u.a. Initiative Regenbogen, „glücklose Schwangerschaft", „verwaiste Eltern“). 
Termination of pregnancy (TOP) Ein Abbruch der Schwangerschaft ist unter Berücksichtigung der gegenwärtigen und zukünftigen Lebensverhältnisse der Schwangeren nach ärztlicher Erkenntnis angezeigt, um eine Gefahr für das Leben oder die Gefahr einer schwerwiegenden Beeinträchtigung des körperlichen oder seelischen $\mathrm{Ge}$ sundheitszustandes der Schwangeren abzuwenden - sofern dies nicht auf eine andere für sie zumutbare Weise geschehen kann.

Rechtliche Grundlagen Das zum 1.1.2010 in Kraft getretene Gesetz zur Änderung des Schwangerschaftskonfliktgesetzes (SchKG) umfasst folgende Veränderungen:

- Die Schwangere erhält mehr Beratung im Zusammenhang mit der Diagnose einer Erkrankung des Kindes, was sowohl medizinische als auch psychosoziale Aspekte angeht.

- Sie muss durch den Arzt obligatorisch darauf hingewiesen werden, dass sie ein Recht auf Beratung durch psychosoziale Beratungsstellen hat.

- Zwischen der Diagnose bzw. Beratung und der Ausstellung der Indikationsbescheinigung müssen mindestens 3 Tage vergehen (Zeit des Überdenkens).

- Nur bei „gegenwärtiger erheblicher Gefahr für Leib und Leben der Schwangeren“ (z. B. bei septischem Abort) gelten diese Fristen nicht.

Nach ausführlicher interdisziplinärer Beratung über das zu erwartende Krankheitsbild wird der Schwangeren unabhängig von ihrer Entscheidung psychosoziale Begleitung angeboten und nahegelegt.

Auch für Eltern, die ein Baby durch einen Schwangerschaftsabbruch nach medizinischer Indikation verloren haben, besteht die Notwendigkeit der Trauerbewältigung.

\section{Entbindung bei Risikoschwangerschaft}

Am besten im Perinatalzentrum Cemäß Mutterschaftsrichtlinie soll der betreuende Arzt die Schwangere auch „bei der Wahl der Entbindungsklinik unter dem Gesichtspunkt beraten, dass die Klinik über die nötigen personellen und apparativen Möglichkeiten zur Betreuung von Risikogeburten und/oder Risikokindern verfügt." [1]

- An den perinatologischen Zentren gibt es etablierte Therapieregime, um Schwangere kompetent und umfassend begleiten können.

Frühgeburtlichkeit Eine geplante vorzeitige Entbindung kann maternal (schwere Erkrankungen) oder fetal begründet sein. Mithilfe der differenzierten Sonografie lassen sich mögliche Verschlechterungen des fetalen Zustandes z.B. bei Plazentainsuffizienz erfassen und eine Schwangerschaftsprolongation gegen eine vorzeitige Entbindung abwägen. Für die Evaluation des "fetal wellbeing“ ist v.a. ein Doppler der fetalen Herz-Kreislaufzirkulation über die präkardialen Venen (z.B. Ductus venosus) und die postkardialen Arterien (Aa. cerebri mediae) aussagekräftig. Es ist Gegenstand laufender und noch zu initiierender Studien, die Frage der geringeren Langzeitmorbidität von Frühgeburtlichkeit versus intrauterinem Abwarten zu beantworten [9].

Risiken einer Ultraschalluntersuchung Für die biologische Wirkung des diagnostischen Ultraschalls wird auf die Stellungnahme der EFSUMB (European Federation of Societies for Ultrasound in Medicin and Biology) verwiesen. Zudem wird empfohlen, die Ultraschalluntersuchung so kurz wie möglich zu halten („As Low As Reasonably Achievable“ - ALARA-Prinzip) [10].

Fazit Jede Schwangerschaft birgt ein gewisses Risiko, ein behindertes oder krankes Kind zu bekommen. Bei einigen Frauen ist die Gefahr jedoch überdurchschnittlich hoch. Mithilfe der Pränataldiagnostik lassen sich viele Auffälligkeiten oder Behinderungen schon während der Schwangerschaft erkennen. Diese können z. T. intrauterin behandelt werden. Zur Verminderung von Morbidität (und Mortalität) kann eine vorzeitige Entbindung indiziert sein. Manchmal muss man die Eltern auch auf die Geburt eines schwerkranken oder toten Kindes vorbereiten. Ein Schwangerschaftsabbruch ist immer die letzte Option.

Erfreulicherweise können aber durch die komplexen pränatalen Untersuchungen die Sorgen der Schwangeren meist ausgeräumt werden. Etwa $96 \%$ aller Kinder kommen gesund zur Welt - manche allerdings zu früh. 


\section{Kernaussagen}

- Etwa 4\% aller Kinder haben angeborene Erkrankungen. Außerhalb spezialisierter Pränatalambulanzen können diese bei den frauenärztlichen Ultraschalluntersuchungen nur teilweise erkannt werden.

- Die häufigsten Fehlbildungen betreffen das Herz, die Nieren und ableitenden Harnwege sowie die Chromosomen.

- Ist eine kindliche Fehlbildung/Erkrankung vor der Geburt bekannt, kann dies die Versorgung und den Lebensstart eines Neugeborenen deutlich verbessern.

- Vor PND sollte beraten werden über:

$\triangleright$ Ziel, Risiko und Grenzen der Untersuchungen - darunter auch pränatal nicht erfassbare Störungen sowie die Sicherheit des Ergebnisses

$\triangleright$ Art und Schweregrad möglicher bzw. vermuteter Störungen

$\triangleright$ Optionen bei pathologischem Befund

$\triangleright$ psychisches und ethisches Konfliktpotenzial

$\triangleright$ Alternativen zur weiterführenden Diagnostik und Möglichkeiten einer weitergehenden psychosozialen Beratung sowie Inanspruchnahme von Hilfsangeboten

- Mithilfe der differenzierten Ultraschalldiagnostik kann man

$\triangleright$ die Versorgungssituation des Ungeborenen bei vorbestehenden oder schwangerschaftsbedingten Erkrankungen der Mutter beurteilen,

$\triangleright$ eine Gefährdung des Feten rechtzeitig erkennen und

$\triangleright$ ggf. durch eine vorzeitige, rechtzeitige Entbindung oder eine intrauterine Therapie Schaden abwenden.

- Ist eine kindliche Erkrankung oder Fehlbildung zu erwarten, ermöglichen einfühlsame fachübergreifende Beratungen und Begleitangebote den werdenden Eltern, sich auf die Geburt ihres kranken Kindes vorzubereiten.
- Das pränatale Wissen um Erkrankungen, die nicht mit dem Leben vereinbar sind (z. B. Anenzephalus, Trisomie 13, 18),

$\triangleright$ ermöglicht den Eltern das Abschiednehmen vom Kind und eine nachgeburtliche Sterbebegleitung,

$\triangleright$ vermeidet unnötige kinderärztliche Eingriffe und damit eine traumatisierende Trennung der Mutter von ihrem Kind,

$\triangleright$ vermeidet unnötige operative geburtshilfliche Eingriffe (z.B. Kaiserschnitt) und erhöht die Chancen für eine weitere Schwangerschaft.

- Ein Schwangerschaftsabbruch ist bei entsprechender Entscheidung der Schwangeren gesetzlich erlaubt, wenn

$\triangleright$ eine schwere Behinderung des Kindes zu erwarten ist,

$\triangleright$ dadurch die Gefahr einer schwerwiegenden Beeinträchtigung des körperlichen oder seelischen Gesundheitszustandes der Schwangeren besteht und

$\triangleright$ diese Gefahr nicht auf andere für sie zumutbare Weise abgewendet werden kann.

- Kein Arzt kann jedoch zur Durchführung eines Schwangerschaftsabbruchs gezwungen werden.

- Auch ein unauffälliges Untersuchungsergebnis garantiert kein gesundes Kind.

- Maternales Alter als alleinige Indikation für ein Screening auf Chromosomenfehler ist inakzeptabel, da die Falschpositivrate bei $25 \%$ liegt.

- Nur interdisziplinäre Zusammenarbeit verbessert das neonatale und mütterliche Outcome.

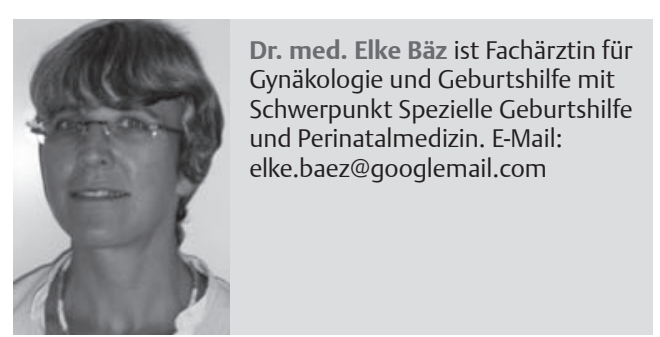

Dr. med. Beatrix Brinckwirth praktiziert als niedergelassene Frauenärztin in Freiburg. Sie ist spezialisiert auf Pränatale Medizin und Ultraschalldiagnostik. E-Mail: info@praenatal-suedwest.de

Interessenkonflikt Die Autoren erklären, dass keine Interessenkonflikte vorliegen.

Beitrag online zu finden unter http://dx.doi. org/10.1055/s-0032-1325309

\section{Literatur}

1 Gemeinsamer Bundesausschuss (G-BA). Richtlinien des Bundesausschusses der Ärzte und Krankenkassen über die ärztliche Betreuung während der Schwangerschaft und nach der Entbindung („Mutterschafts-Richtlinien“) in der Fassung vom 10.12.1985, zuletzt geändert am 19.5.2011, veröffentlicht im Bundesanzeiger Nr. 124, S. 2894 in Kraft getreten am 19.8.2011. Im Internet: www.g-ba.de/downloads/62-492-591/Mu-RL_2011-12-15.pdf

2 Gemeinsamer Bundesausschuss (G-BA). Vorläufiger Bericht zum Beratungsverfahren gemäß $\S$ 135 Abs. 1 SGB V i.V.m. § 196 der Reichsversicherungsordnung (RVO) Ultraschallscreening in der Schwangerschaft Stand: 16.9.2010 Beschluss noch nicht in Kraft.

3 Snijders RJ, Noble P, Sebire N et al. UK multicentre project on assessment of risk of trisomy 21 by maternal age and fetal nuchal-translucency thickness at 10-14 weeks of gestation. Fetal Medicine Foundation First Trimester Screening Group. Lancet 1998; 352: 343-346

4 Nicolaides KH. A model for a new pyramid of prenatal care based on the 11 to 13 weeks' assessment. Prenat Diagn 2011; 31: 3-6

5 Baez E et al. Ersttrimester-Sonografie. Geburtsh Frauenheilk 2002; 62: Rn. 81-Rn. 100 (Refresher)

6 ACOG Practice Bulletin No. 77: screening for fetal chromosomal abnormalities. Obstet Gynecol 2007; 109: 217-227

7 Deprest J, Jani J, Gratacos E et al. Fetal intervention for congenital diaphragmatic hernia: the European experience. Semin Perinatol 2005; 29: 94-103

8 Adzick NS, Thom EA, Spong CY et al. A Randomized Trial of Prenatal versus Postnatal Repair of Myelomeningocele. N Engl J Med 2011; 364: 993-1004

9 Baschat AA. Neurodevelopment following fetal growth restriction and its relationship with antepartum parameters of placental dysfunction. Ultrasound Obstet Gynecol 2011; 37: 501-514

10 Rott HD. EFSUMB-Statement über klinische Sicherheit der Ultraschalldiagnostik. Ultraschall Med 1998; $19: 192$ 